Incidence and impact of chronic lung allograft dysfunction after lung transplantation - single-center 14-year experience

\title{
Nykänen, Antti
}

2020-05-03

Nykänen , A , Raivio , P , Peräkylä , L , Stark , C , Huuskonen , A , Lemström , K, Halme , M \& Hämmäinen , P 2020 , ' Incidence and impact of chronic lung allograft dysfunction after lung transplantation - single-center 14-year experience ' , Scandinavian Cardiovascular Journal, vol. 54 , no. 3 , pp. 192-199 . https://doi.org/10.1080/14017431.2020.1726444

http://hdl.handle.net/10138/327662

https://doi.org/10.1080/14017431.2020.1726444

acceptedVersion

Downloaded from Helda, University of Helsinki institutional repository.

This is an electronic reprint of the original article.

This reprint may differ from the original in pagination and typographic detail.

Please cite the original version. 


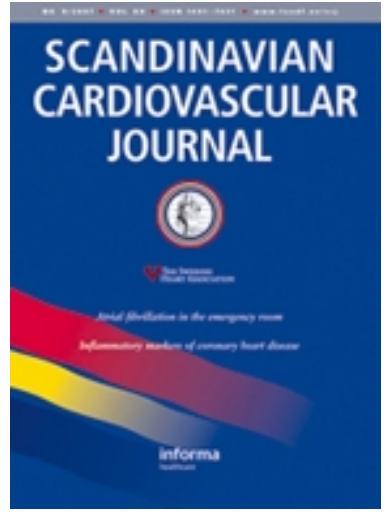

\section{Incidence and impact of chronic lung allograft dysfunction after lung transplantation - single-center 14-year experience}

\begin{tabular}{|c|c|}
\hline Journal: & Scandinavian Cardiovascular Journal \\
\hline Manuscript ID & SCAR-2019-OR-0381.R1 \\
\hline Manuscript Type: & Original Article \\
\hline $\begin{array}{r}\text { Date Submitted by the } \\
\text { Author: }\end{array}$ & $\mathrm{n} / \mathrm{a}$ \\
\hline Complete List of Authors: & $\begin{array}{l}\text { Nykänen, Antti; Helsinki University Hospital, Cardiac Surgery; University } \\
\text { of Helsinki, } \\
\text { Raivio, Peter; Helsinki University Hospital, Cardiac Surgery; University of } \\
\text { Helsinki, } \\
\text { Peräkylä, Laura; Helsinki University Hospital, Cardiac Surgery; University } \\
\text { of Helsinki, } \\
\text { Stark, Christoffer; Helsinki University Hospital, Cardiac Surgery; } \\
\text { University of Helsinki, } \\
\text { Huuskonen, Antti; Helsinki University Hospital, Cardiac Surgery; } \\
\text { University of Helsinki, } \\
\text { Lemström, Karl; Helsinki University Hospital, Cardiac Surgery; University } \\
\text { of Helsinki, } \\
\text { Halme, Maija; Helsinki University Hospital, Pulmonology; University of } \\
\text { Helsinki, } \\
\text { Hämmäinen, Pekka; Helsinki University Hospital, Cardiac Surgery; } \\
\text { University of Helsinki, }\end{array}$ \\
\hline Keywords: & $\begin{array}{l}\text { lung transplantation, end-stage pulmonary disease, chronic lung allograft } \\
\text { dysfunction, chronic rejection, bronchiolitis obliterans syndrome, } \\
\text { restrictive allograft syndrome }\end{array}$ \\
\hline Abstract: & $\begin{array}{l}\text { Objectives. Lung transplantation remains the only available treatment } \\
\text { option for many end-stage lung diseases. We evaluated our long-term } \\
\text { lung transplantation results, and the incidence and impact of chronic } \\
\text { lung allograft dysfunction (CLAD). Design. All adult de novo lung } \\
\text { transplants performed between } 2003 \text { and } 2015(n=175) \text { in a nationwide } \\
\text { single transplant center were retrospectively analyzed. Patients surviving } \\
\text { at least } 90 \text { days with adequate pulmonary function tests available were } \\
\text { evaluated for CLAD ( } n=167, \text { CLAD cohort). Kaplan-Meier survival and } \\
\text { Cox regression analysis were used to evaluate the effect of CLAD. }\end{array}$ \\
\hline
\end{tabular}


Results. Recipient and graft 1-, 5- and 10-year survival estimates for all adult de novo lung transplants were $94 \%, 79 \%$ and $64 \%$, and $93 \%$, $75 \%$ and $59 \%$, respectively. CLAD affected $43 \%$ of CLAD cohort patients, was diagnosed at a median of 2.3 years after transplantation, and impaired recipient $(p=0.03)$ and graft survival $(p=0.001)$ with the most advanced CLAD stage, and restrictive CLAD phenotype, resulting in worst graft survival. CLAD was the primary cause of death in $54 \%$ of all patients, and in $80 \%$ of patients with an established CLAD diagnosis. CLAD, high-risk cytomegalovirus serostatus, and recipient preoperative sensitization increased graft loss hazard ratio in univariate regression analysis. CLAD was the only significant investigated risk factor for graft loss in multivariate regression analysis (hazard ratio $2.77,95 \%$ confidence interval $1.37-5.61, p=0.005$ ). Conclusions. Although very favourable lung transplant patient long-term survival was achieved, CLAD significantly impaired recipient and graft survival. Identification of risk factors and therapeutic options for CLAD may further improve lung transplantation results.

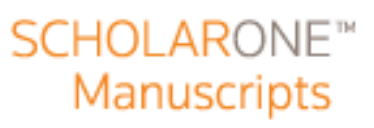




\title{
Incidence and impact of chronic lung allograft dysfunction
}

\section{after lung transplantation - single-center 14-year experience}

\author{
Antti Nykänen, Peter Raivio ${ }^{a}$, Laura Peräkyläa, Christoffer Stark ${ }^{\mathrm{a}}$, Antti Huuskonen ${ }^{\mathrm{a}}$, Karl \\ Lemström $^{\mathrm{a}}$, Maija Halme ${ }^{\mathrm{b}}$, Pekka Hämmäinen ${ }^{\mathrm{a}}$ \\ Affiliations

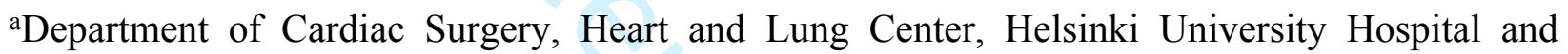 \\ University of Helsinki, Helsinki, Finland; bDepartment of Pulmonology, Heart and Lung Center, \\ Helsinki University Hospital and University of Helsinki, Helsinki, Finland
}

Correspondence to Antti Nykänen, Meilahti Tower Hospital, Haartmaninkatu 4, PL 340, 00029 Helsinki, Finland. Email: Antti.Nykanen@Hus.Fi

Total word count: 5244 


\section{Abstract (250/250 words)}

Objectives. Lung transplantation remains the only available treatment option for many end-stage lung diseases. We evaluated our long-term lung transplantation results, and the incidence and impact of chronic lung allograft dysfunction (CLAD). Design. All adult de novo lung transplants performed between 2003 and $2015(\mathrm{n}=175)$ in a nationwide single transplant center were retrospectively analyzed. Patients surviving at least 90 days with adequate pulmonary function tests available were evaluated for CLAD ( $\mathrm{n}=167$, CLAD cohort). Kaplan-Meier survival and Cox regression analysis were used to evaluate the effect of CLAD. Results. Recipient and graft 1-, 5and 10-year survival estimates for all adult de novo lung transplants were $94 \%, 79 \%$ and $64 \%$, and $93 \%, 75 \%$ and $59 \%$, respectively. CLAD affected $43 \%$ of CLAD cohort patients, was diagnosed at a median of 2.3 years after transplantation, and impaired recipient $(\mathrm{p}=0.03)$ and graft survival ( $\mathrm{p}=0.001)$ with the most advanced CLAD stage, and restrictive CLAD phenotype, resulting in worst graft survival. CLAD was the primary cause of death in $54 \%$ of all patients, and in $80 \%$ of patients with an established CLAD diagnosis. CLAD, high-risk cytomegalovirus serostatus, and recipient preoperative sensitization increased graft loss hazard ratio in univariate regression analysis. CLAD was the only significant investigated risk factor for graft loss in multivariate regression analysis (hazard ratio $2.77,95 \%$ confidence interval $1.37-5.61, \mathrm{p}=0.005$ ). Conclusions. Although very favourable lung transplant patient long-term survival was achieved, CLAD significantly impaired recipient and graft survival. Identification of risk factors and therapeutic options for CLAD may further improve lung transplantation results.

Keywords: Lung transplantation, end-stage pulmonary disease, chronic lung allograft dysfunction, chronic rejection, bronchiolitis obliterans syndrome 


\section{Introduction}

Lung transplantation is an established and often the only available treatment modality for endstage lung disease. The number of lung transplants performed world-wide has been increasing over time with over 4500 adult lung transplantations reported to the International Society for Heart and Lung Transplantation (ISHLT) registry for the year 2016 [1]. Lung transplant results have also gradually improved, and in the ISHLT registry, the median survival of recipients transplanted between 2009 and 6/2016 is 6.5 years [1]. Although short-term results have improved significantly, long-term survival of lung transplant recipients remains suboptimal, and lower than that of other solid organ transplant recipients [2,3].

Chronic lung allograft dysfunction (CLAD) has been identified as the main reason for graft loss 1 year after lung transplantation and it affects $41 \%$ of lung transplant recipients within 5 years [1]. CLAD has been previously known as chronic rejection or bronchiolitis obliterans syndrome (BOS) [4]. The latest consensus statement defines CLAD as a persistent and chronic decline in the function of the transplanted lung in the absence of other specific reasons, and identifies separate CLAD stages according to the degree of lung function decline [5]. Reflecting the evolving knowledge of CLAD, it is currently divided into two main subgroups - BOS that results in an obstructive lung function and restrictive allograft syndrome (RAS) that is characterised by restrictive lung function [5-7]. Diagnosis of CLAD requires persistent forced expiratory volume in 1 second (FEV1) decline to $<80 \%$ of the best postoperative (baseline) FEV1. The golden standard to differentiate BOS and RAS is total lung capacity (TLC) change measured by body plethysmography [5]. Detection of restrictive lung physiology with spirometry can be used for 
CLAD phenotyping if TLC is not available [6,7]. Preventive and therapeutic options for CLAD are currently limited, but re-transplantation may be an option in selective cases [4].

\begin{abstract}
We retrospectively evaluated the long-term results of lung transplantation in our unit - a single nationwide transplant center. During the entire study period a standardized protocol of triple-drug immunosuppression and antimicrobial prophylaxis was used. We also aimed to identify risk factors for graft loss, and specifically focused on the clinical impact of CLAD. Subtypes of CLAD were evaluated by spirometric criteria $[6,7]$ as TLC measurement with body plethysmography was not routinely used during the study period.
\end{abstract}




\section{Materials and methods}

\section{Study design and population}

We retrospectively analyzed all consecutive 175 adult (recipient $>18$ years) de novo lung transplantations performed in a nationwide single organ transplant center (Helsinki University Hospital, Helsinki, Finland) between January 2003 and December 2015. Patients were followed up until March 2017, and the median follow-up time was 6.0 years (2189 days), ranging from 1.3 to 14.0 years. Graft and recipient survival was determined for the whole study population $(\mathrm{n}=175)$, and patients who survived for at least 90 days, and had adequate pulmonary function tests available, were evaluated for CLAD ( $\mathrm{n}=167$, CLAD patient cohort). Patient information was collected from electronic patient records, and from donor and recipient data submitted to the Collaborative Transplant Study Registry [3]. The study was reviewed and approved by The Institutional Review Board of the Helsinki University Hospital.

\section{Surgical and patient management factors}

Almost all transplantations were performed through a clamshell incision (96\%) using cardiopulmonary bypass $(99 \%)$ and were bilateral lung transplants (97\%). Perioperative and postoperative management was guided by a standardized written institutional protocol. Standard triple-drug primary immunosuppression consisted of a calcineurin inhibitor (100\% of patients), mycophenolate mofetil ( $98 \%$ of patients), and corticosteroids ( $98 \%$ of patients), and only $1 \%$ of patients received induction therapy. Standard perioperative antimicrobial prophylaxis consisted of intravenous ceftazidime or meropenem, and caspofungin, and postoperative inhalation of nebulized amphotericin B. Antiviral prophylaxis was achieved with acyclovir (3 months for 
cytomegalovirus donor-/recipient-) or valganciclovir (6 months for CMV D+/R+ and D-/R+, 12 months for CMV D+/R-). Trimethoprim/sulfamethoxazole was the primary prophylaxis for pneumocystis jirovecii. Recipients routinely received proton-pump inhibitors. Azithromycin was started for patients with early signs of decreased pulmonary function or with BOS risk factors such as recurrent acute rejections, BAL neutrophilia or gastroesophageal reflux. All CLAD patients received azithromycin. The median for azithromycin initiation was 1.1 years after transplantation. Fifty-nine percent of CLAD patients were treated with azithromycin before CLAD diagnosis whereas $41 \%$ of CLAD patients were initiated with azithromycin only after CLAD diagnosis. Transbronchial surveillance biopsies were obtained 1, 2, 4, 6, 9 and 12 months after transplantation, and graded according to ISHLT classification [8]. Bronchoalveolar lavage sample and spirometry were performed during the same intervals, and yearly thereafter. TLC measurement with body plethysmography was not routinely used during the study period. Donor, recipient, and operative characteristics are given in Table 1. Criteria for ideal and extended donors were used as described previously [9].

\section{CLAD diagnosis and staging}

CLAD was evaluated using the lung function measurements recorded with spirometry during follow-up visits. All patients surviving $>90$ days who had pulmonary function tests available were included in CLAD analysis ( $\mathrm{n}=167$, Figure 1). CLAD was defined as a persistent FEV1 or forced vital capacity (FVC) decline to $\leq 80 \%$ of the best postoperative (baseline) values in the absence of other specific reasons for the lung function decline $[5,10]$. CLAD onset was defined to occur at the first time for the sustained pulmonary function decrease below the threshold [5]. CLAD was further divided into stages as suggested by the latest consensus document (CLAD Stage 1 FEV1 
$>65-80 \%$ FEV1 baseline; CLAD Stage 2 FEV1 >50-65\% FEV1 baseline; CLAD Stage 3 FEV1

$>35-50 \%$ FEV1 baseline; and CLAD 4 FEV1 $\leq 35 \%$ FEV1 baseline) [5]. As body plethysmography TLC measurement - the golden standard for BOS and RAS differentiation [6] was not routinely used during the study period, CLAD subtypes were evaluated by spirometric criteria for restrictive pulmonary function [7]. Patients whose FVC declined at CLAD onset to $\leq 80 \%$ of their baseline FVC (FVC value at the time of the best postoperative FEV1) [7] were classified as RAS, and other CLAD patients were classified as BOS.

\section{Statistical analysis}

All statistical analyses were performed using the SPSS 25.0 (IBM Corp., Armonk, NY) software. Fisher's exact test was used to compare categorical variables and Student's t-test was used to compare parametric continuous variables. For survival analyses, data is presented as Kaplan-Meier curves and analyzed with the log-rank test. $\mathrm{P}<0.05$ was considered statistically significant. Cox univariate regression analysis was used to determine the hazard ratio of covariates for graft survival. All covariates with a p-value $<0.20$ in univariate analysis were included in a Cox multivariate regression analysis. 


\section{Results \\ Graft and recipient survival of all de novo lung transplants}

One-hundred-seventy-five adult de novo lung transplantations were performed between January 2003 and December 2015, and pulmonary function data were available for CLAD determination in 167 patients (Figure 1). Recipient and graft 1-, 5- and 10-year survival estimates for all adult de novo lung transplants $(\mathrm{n}=175)$ were $94 \%, 79 \%$, and $64 \%$, and $93 \%, 75 \%$, and $59 \%$, respectively (Figure 2A). In contrast, CLAD and re-transplantation -free survival estimates (survival with a well-functioning primary graft) for all adult de novo lung transplants were $86 \%, 52 \%$, and $28 \%$ for 1,5 , and 10 years, respectively (Figure 2A).

\section{CLAD diagnosis}

One-hundred-sixty-seven patients survived at least 90 days and had adequate pulmonary function measurements for the determination of possible CLAD (Figure 1, $\mathrm{n}=167$ ). Baseline donor and recipient characteristics of the CLAD patient cohort are summarized in Table 1. CLAD was diagnosed in $43 \%(\mathrm{n}=71)$ of the CLAD cohort patients with a median diagnosis at 893 days $(2.3$ years) after transplantation (Table 2). Most CLAD patients were categorized as CLAD stage 1 or 2 whereas more advanced CLAD stage was uncommon at the time of CLAD onset (Table 2). CLAD was subclassified as BOS in $75 \%(n=53)$ and as RAS in $25 \%(n=18)$ using spirometric criteria for restrictive pulmonary function (Table 2).

\section{Effect of CLAD on outcome}


CLAD was the primary cause of death in $54 \%$ of the whole CLAD patient cohort, and in $80 \%$ of patients diagnosed with CLAD (Table 3). The most common primary cause of death in non-CLAD patients was malignancy (42\%). Eight patients (5\% of all CLAD cohort patients, $11 \%$ of patients with CLAD diagnosis) were re-transplanted due to CLAD. Recipient ( $p=0.03$, Figure $2 \mathrm{~B})$ and graft survival ( $p=0.001$, Figure $2 C$ ) was significantly impaired in patients with CLAD, and patients with the most advanced CLAD stage at CLAD onset had the worst graft survival (Figure 2E). Patients with RAS phenotype had worse graft survival than patients with BOS phenotype $(\mathrm{p}<0.05$, Figure $2 \mathrm{~F})$.

\section{Univariate regression analysis on graft loss}

CLAD significantly increased the hazard ratio for graft loss in a univariate regression analysis $(p=0.002$, Table 4). Other significant covariates for graft loss were high risk CMV serostatus $(\mathrm{CMV} D+/ \mathrm{R}-, \mathrm{p}=0.02)$ and recipient preoperative sensitization $(\mathrm{p}=0.03)$, whereas increased donor age $(p=0.10)$, single lung transplant $(p=0.17)$ and extended criteria donor $(p=0.17)$ were associated only with a trend towards increased hazard ratio for graft loss. Higher best postoperative FEV1 $(p=0.003)$ and FVC $(p=0.002)$ values had a significantly decreased hazard ratio for graft loss.

\section{Multivariate regression analysis on graft loss}

All covariates with a p-value $<0.20$ in the univariate analysis were included in multivariate regression analysis (Table 5). Of the investigated factors, CLAD was the only covariate that significantly increased the hazard ratio for graft loss (hazard ratio $2.77,95 \%$ confidence interval $1.37-5.61, \mathrm{p}=0.005)$. 


\section{Discussion}

We retrospectively evaluated our long-term lung transplantation results. A favourable long-term recipient survival was achieved. However, CLAD was as a major obstacle for long-term survival, as it was the most common primary cause of death, CLAD significantly impaired recipient and graft survival, and it was the strongest covariate to increase the hazard ratio for graft loss in univariate and multivariate regression analysis.

\section{Comparison of lung transplant long-term results}

The 5-year survival estimate of our adult primary lung transplant cohort transplanted between 2003 and 2015 was $79 \%$. It is favourable compared to the survival of the latest transplantation cohort (transplants between 2009 and 6/2016) reported to the ISHLT registry [1]. Although caution is warranted when interpreting the long-term survival estimates, and when comparing single center data to registry data, some donor, recipient, and patient management -related reasons may explain the favourable recipient survival of our patient cohort. First, most of our recipients received a double-lung transplant and single lung transplantation was performed only for $4 \%$ of recipients. In the ISHLT registry, single lung recipients have impaired long-term survival, and about $20 \%$ of lung transplants performed are single-lung transplantations [1] which is a significantly higher proportion than in our patient cohort. However, the evidence for the superiority of bilateral lung transplantation is not completely clear, and single-lung transplantation particularly in selective cases, or as a staged strategy, may lead to good results [11]. Second, infection was the primary cause of death in only $11 \%$ of our patients whereas infection has been reported as the known cause of death in up to $36 \%$ of lung transplant recipients early (31 days - 1 year) after transplantation [1]. Also, the incidence of airway anastomosis problems was low as no anastomosis dehiscence 
was detected, no airway stents were applied, and balloon dilatation was performed in only 5 patients $(3 \%)$. Previously, we encountered a significant amount of invasive infections that were possibly related to overimmunosuppression [12]. During the present study period, antithymocyte globulin induction was not routinely administered anymore, and close calcineurin inhibitor concentration monitoring, and standardized perioperative bacterial and fungal prophylaxis, was used [12]. Therefore, the balance of immunosuppression and antimicrobial prophylaxis in our patient cohort has probably been improved. Third, eight recipients $(5 \%$ of all patients) were retransplanted due to CLAD. Re-transplantation results have improved over time [13], and most likely the outcome of our patients re-transplanted due to CLAD would have been dismal without the possibility for a second transplant. Finally, it is possible that donor and recipient characteristics of our patient cohort affected the results. However, the median age of our donors was 46 years compared to 38 years for the donors reported to the ISHLT for the year 2012 [14], and half of our donors were extended criteria donors. The age of our recipients was comparable to the ISHLT registry. Furthermore, the proportion of transplants performed for interstitial lung disease was higher ( $50 \%$ of our primary indications) than what is reported to the ISHLT [14], and patients with this indication generally have worse survival than patients transplanted for other indications. Also, as about $10 \%$ of our recipients were bridged to transplant with ECMO or invasive ventilation, it is unlikely that significant selection bias for donors or recipients explains our results.

\section{Significance of CLAD for lung transplant recipient morbidity and mortality}

CLAD has been repeatedly identified as the main obstacle for long-term survival of lung transplant recipients $[1,4,5]$, In our patient cohort, CLAD affected $43 \%$ of the recipients and was the primary cause of death in $54 \%$ of the patients. It significantly impaired recipient and graft survival, and 
resulted in re-transplantation in $11 \%$ of the patients who had developed CLAD. Moreover, although 10-year recipient survival for all adult de novo transplants was $64 \%$, only $24 \%$ of the recipients were alive without re-transplantation, and with a well-functioning primary lung graft at 10 years. Therefore, CLAD was clearly the main cause of morbidity and mortality also in our patient cohort.

\section{Survival after CLAD onset}

Although the clinical impact of CLAD was clear, the median survival of patients affected with CLAD was 7.0 years after the onset of the disease which is longer than what is generally reported for BOS (3-5 years) or RAS (1-1.5 years) [4]. As our CLAD incidence was very similar to the ISHLT registry [1], it is possible that some factors protected against the detrimental effects of CLAD in our cohort. Our preference for double-lung transplantations may have increased the pulmonary reserve once CLAD occurred [15], and re-transplantations most probably improved survival of patients affected by CLAD substantially. Also, all CLAD patients were treated with azithromycin which may have slowed down the pulmonary function decline [16].

\section{CLAD subtypes}

Although significant advances have been made in characterizing CLAD, the pathophysiological mechanisms are not entirely understood, and preventive and treatment options currently are limited. Importantly, specific CLAD subtypes - BOS and RAS - have been identified. RAS has an even worse clinical outcome than BOS with a median survival limited to only 6-18 months after diagnosis. Despite the dismal clinical course of RAS, information on predisposing factors, 
pathogenesis and clinical course is currently very limited [17]. It is possible that detailed characterization of CLAD phenotypes may result in tailored preventive and therapeutic options. We also found that patients with restrictive CLAD phenotype had worse graft survival than patients with obstructive phenotype. As TLC measurement with body plethysmography - the golden standard also endorsed by the latest CLAD and RAS consensus statements $[5,6]$ - was not routinely used during the study period, we used spirometric criteria described by Todd et al. to differentiate BOS and RAS [7].

\section{CMV and recipient sensitization as risk factors for graft loss}

In addition to CLAD, we found that high risk CMV serostatus, and recipient preoperative sensitization, both increased the hazard ratio for graft loss in univariate analysis. CMV D+/Rserotype is a known risk factor for early and late mortality [1]. However, prophylaxis for the highrisk serotypes with 12-month valganciclovir similar to our study cohort, followed by close CMV monitoring with pre-emptive treatment strategies, reduces the incidence of CMV disease [18]. Higher preoperative PRA percentage is also an established risk factor for mortality [1] but tailored perioperative desensitization strategies may be used to safely transplant even highly sensitized recipients [19].

\section{Limitations of the current study}

Several limitations of the study must be considered when interpreting the results. Due to the retrospective nature of the study, it is possible that changes in clinical practise have occurred over time, and differences in donor and recipient characteristic, and transplant volume, over time may 
bias the incidence of time-dependent events. These likely include increased availability and use of ECMO, and more liberal donor and recipient criteria over time. Similarly, results from a singlecenter transplant center with a confined population area may be difficult to generalize. This generalization may be also affected by the transplant indication profile of our patient cohort as the proportion of ILD patients is higher, and the proportion of chronic obstructive pulmonary disease or cystic fibrosis is lower, than what is reported to the ISHLT registry [1]. Finally, due to reasons described above, we evaluated CLAD subtypes by spirometry, and not TLC measurement which is presently recommended to differentiate BOS and RAS [6].

In conclusion, retrospective analysis of our lung transplantation results demonstrates favourable long-term patient survival. Importantly, CLAD was the most significant risk factor for impaired recipient and graft survival. Therefore, in the future, identification of risk factors of CLAD and development of therapeutic options for CLAD may further improve lung transplantation results. 


\section{Acknowledgements}

We are grateful for the invaluable help of transplant coordinators Marja-Liisa Hellstedt, Catharina Yesil, and Sini Puputti, in patient management and data collection.

\section{Funding details}

This work was supported by the The Jalmari and Rauha Ahokas Foundation and The Sigrid Juselius Foundation.

\section{Declaration of interest statement}

The authors report no conflict of interest. 


\section{References}

1. Chambers DC, Cherikh WS, Goldfarb SB, et al. The International Thoracic Organ Transplant Registry of the International Society for Heart and Lung Transplantation: Thirty-fifth adult lung and heart-lung transplant report-2018; Focus theme: Multiorgan Transplantation. J Heart Lung Transplant. 2018 Oct;37(10):1169-1183.

2. Lodhi SA, Lamb KE, Meier-Kriesche HU. Solid organ allograft survival improvement in the United States: the long-term does not mirror the dramatic short-term success. Am J Transplant. 2011 Jun;11(6):1226-35.

3. Opelz G, Dohler B, Ruhenstroth A, et al. The collaborative transplant study registry. Transplant Rev (Orlando). 2013 Apr;27(2):43-5.

4. Verleden SE, Vos R, Verleden GM. Chronic lung allograft dysfunction: light at the end of the tunnel? Current opinion in organ transplantation. 2019 Jun;24(3):318-323.

5. Verleden GM, Glanville AR, Lease ED, et al. Chronic lung allograft dysfunction: Definition, diagnostic criteria, and approaches to treatment-A consensus report from the Pulmonary Council of the ISHLT. J Heart Lung Transplant. 2019 May;38(5):493-503.

6. Glanville AR, Verleden GM, Todd JL, et al. Chronic lung allograft dysfunction: Definition and update of restrictive allograft syndrome-A consensus report from the Pulmonary Council of the ISHLT. J Heart Lung Transplant. 2019 May;38(5):483-492.

7. Todd JL, Jain R, Pavlisko EN, et al. Impact of forced vital capacity loss on survival after the onset of chronic lung allograft dysfunction. Am J Respir Crit Care Med. 2014 Jan 15;189(2):159-66.

8. Stewart S, Fishbein MC, Snell GI, et al. Revision of the 1996 working formulation for the standardization of nomenclature in the diagnosis of lung rejection. J Heart Lung Transplant. 2007 Dec;26(12):1229-42.

9. Orens JB, Boehler A, de Perrot $\mathrm{M}$, et al. A review of lung transplant donor acceptability criteria. J Heart Lung Transplant. 2003 Nov;22(11):1183-200.

10. Verleden GM, Raghu G, Meyer KC, et al. A new classification system for chronic lung allograft dysfunction. J Heart Lung Transplant. 2014 Feb;33(2):127-33.

11. Subramanian MP, Meyers BF. Bilateral versus single lung transplantation: are two lungs better than one? Journal of thoracic disease. $2018 \mathrm{Jul} ; 10$ (7):4588-4601.

12. Eriksson $\mathrm{M}$, Lemstrom $\mathrm{K}$, Suojaranta-Ylinen $\mathrm{R}$, et al. Control of early Aspergillus mortality after lung transplantation: outcome and risk factors. Transplant Proc. 2010 Dec;42(10):4459-64.

13. Lindstedt $\mathrm{S}$, Dellgren $\mathrm{G}$, Iversen $\mathrm{M}$, et al. Pulmonary retransplantation in the Nordic countries. The Annals of thoracic surgery. 2015 May;99(5):1781-7.

14. Yusen RD, Christie JD, Edwards LB, et al. The Registry of the International Society for Heart and Lung Transplantation: Thirtieth Adult Lung and Heart-Lung Transplant Report--2013; focus theme: age. J Heart Lung Transplant. 2013 Oct;32(10):965-78.

15. Mason DP, Rajeswaran J, Li L, et al. Effect of changes in postoperative spirometry on survival after lung transplantation. J Thorac Cardiovasc Surg. 2012 Jul;144(1):197-203.

16. Corris PA, Ryan VA, Small T, et al. A randomised controlled trial of azithromycin therapy in bronchiolitis obliterans syndrome (BOS) post lung transplantation. Thorax. 2015 May;70(5):44250.

17. Verleden SE, Ruttens D, Vandermeulen E, et al. Predictors of survival in restrictive chronic lung allograft dysfunction after lung transplantation. J Heart Lung Transplant. 2016 Apr 16.

18. Chang A, Musk M, Lavender $\mathrm{M}$, et al. Cytomegalovirus viremia in lung transplantation during and after prophylaxis. Transpl Infect Dis. 2019 Jun;21(3):e13069. 
19. Tinckam KJ, Keshavjee S, Chaparro C, et al. Survival in sensitized lung transplant recipients with perioperative desensitization. Am J Transplant. 2015 Feb;15(2):417-26. 
Table 1. CLAD cohort characteristics.

\begin{tabular}{|c|c|c|c|c|}
\hline Variables & $\begin{array}{c}\text { All patients } \\
n=167\end{array}$ & $\begin{array}{c}\text { No CLAD } \\
n=96(57 \%)\end{array}$ & $\begin{array}{c}C L A D \\
n=71(43 \%)\end{array}$ & $\begin{array}{l}\text { p-value } \\
\text { No CLAD } \\
\text { vs CLAD }\end{array}$ \\
\hline \multicolumn{5}{|l|}{ Transplant information } \\
\hline Bilateral lung transplant, n (\%) & $161(96)$ & 95 (99) & $66(93)$ & 0.08 \\
\hline Single lung transplant, $\mathrm{n}(\%)$ & $6(4)$ & $1(1)$ & $5(7)$ & 0.08 \\
\hline \multicolumn{5}{|l|}{ Perioperative support } \\
\hline Pre-operative ECMO, n (\%) & $15(9)$ & $9(9)$ & $6(9)$ & 1 \\
\hline Preoperative invasive ventilation, $\mathrm{n}(\%)$ & $13(8)$ & $7(7)$ & $6(9)$ & 0.78 \\
\hline Postoperative ECMO, n (\%) & $1(1)$ & $0(0)$ & $1(1)$ & 0.43 \\
\hline Transplant urgency & & & & 0.25 \\
\hline Normal, n (\%) & $141(84)$ & $78(80)$ & $63(89)$ & \\
\hline Urgent, n (\%) & $11(7)$ & $9(10)$ & $2(5)$ & \\
\hline Super Urgent, n (\%) & $15(9)$ & $9(10)$ & $6(6)$ & \\
\hline Primary diagnosis & & & & 0.045 \\
\hline Emphysema/COPD, n (\%) & $27(16)$ & $12(13)$ & $15(21)$ & \\
\hline Cystic fibrosis, n (\%) & $12(7)$ & $11(11.5)$ & $1(1)$ & \\
\hline Interstitial lung disease, $\mathrm{n}(\%)$ & $84(50)$ & $50(52)$ & $34(48)$ & \\
\hline Alpha-1 -antitrypsin deficiency, n (\%) & $17(10)$ & $11(11.5)$ & $6(9)$ & \\
\hline Pulmonary hypertension, $\mathrm{n}(\%)$ & $14(9)$ & $5(5)$ & $9(12)$ & \\
\hline Other, n (\%) & $13(8)$ & $7(7)$ & $6(9)$ & \\
\hline \multicolumn{5}{|l|}{ Recipient characteristics } \\
\hline Recipient age at transplant, median (IQR) & $56(16)$ & $56(19)$ & $58(15)$ & 0.38 \\
\hline Recipient sex female, $\mathrm{n}(\%)$ & $68(41)$ & $38(40)$ & $30(42)$ & 0.75 \\
\hline Recipient history of smoking, n (\%) & $100(60)$ & $55(57)$ & $45(63)$ & 0.52 \\
\hline PRA positive before transplantation, $n(\%)$ & $50(30)$ & $31(32)$ & $19(29)$ & 0.73 \\
\hline$\geq 4$ HLA mismatches in HLA-A, B or DR & $71(43)$ & $39(41)$ & $32(45)$ & 0.64 \\
\hline \multicolumn{5}{|l|}{ Primary immunosuppression } \\
\hline Cyclosporine A, n (\%) & $147(88)$ & $80(83)$ & $67(94)$ & 0.03 \\
\hline Tacrolimus, n (\%) & $20(12)$ & $16(17)$ & $4(6)$ & 0.03 \\
\hline Any azithromycin use after transplantation & $147(88)$ & $76(79)$ & $71(100)$ & $<0.001$ \\
\hline \multicolumn{5}{|l|}{ Donor characteristics } \\
\hline Donor age, median (IQR) & $46(21)$ & $45.5(26)$ & $47(15)$ & 0.07 \\
\hline Donor sex female, n (\%) & $73(44)$ & $55(57)$ & $39(55)$ & 0.88 \\
\hline Total ischemia time minutes, median (IQR) & $259(96)$ & $262(95)$ & $254(107)$ & 0.19 \\
\hline Extended criteria donor, $\mathrm{n}(\%)$ & $86(51)$ & $47(49)$ & $39(55)$ & 0.53 \\
\hline CMV D+/R-, n (\%) & $18(11)$ & $8(5)$ & $10(14)$ & 0.31 \\
\hline \multicolumn{5}{|l|}{ Biopsy-proven rejections } \\
\hline Any abnormal finding $<1$ year, $\mathrm{n}(\%)$ & $97(58)$ & $57(59)$ & $40(57)$ & 0.87 \\
\hline Any acute rejection < 1 year, $\mathrm{n}(\%)$ & $90(54)$ & $53(55)$ & $37(53)$ & 0.88 \\
\hline$\geq A 2$ grade $<1$ year, $n(\%)$ & $47(28)$ & $31(32)$ & $16(23)$ & 0.22 \\
\hline Any airway inflammation < 1 year, $\mathrm{n}(\%)$ & $38(23)$ & $25(26)$ & $13(19)$ & 0.35 \\
\hline$\geq \mathrm{B} 2$ grade $<1$ year, $\mathrm{n}(\%)$ & $8(5)$ & $7(7)$ & $1(1)$ & 0.14 \\
\hline
\end{tabular}

Data are expressed as $n$ (\% of the corresponding patient group) and analyzed by Fisher's Exact Test for 
categoric variables, or expressed as median (IQR, interquartile range) and analyzed by Student's t-test for parametric continuous variables.

CLAD: chronic lung allograft dysfunction; CMV: cytomegalovirus; COPD: chronic obstructive pulmonary disease; D: donor; ECMO: extra-corporeal membrane oxygenation; HLA: human leukocyte antigen; PRA: panel reactive antibody; R: recipient. 
Table 2. Pulmonary function and CLAD of CLAD cohort patients

\begin{tabular}{|c|c|c|c|c|}
\hline Variables & $\begin{array}{c}\text { All patients } \\
\mathrm{n}=167\end{array}$ & $\begin{array}{c}\text { No CLAD } \\
\mathrm{n}=96(57 \%)\end{array}$ & $\begin{array}{c}\text { CLAD } \\
\mathrm{n}=71(43 \%)\end{array}$ & $\begin{array}{l}\text { p-value } \\
\text { No CLAD } \\
\text { vs CLAD }\end{array}$ \\
\hline CLAD diagnosis days after transplant, median (IQR) & & & $839(952)$ & \\
\hline \multicolumn{5}{|l|}{ CLAD stage at CLAD onset } \\
\hline CLAD 1 (FEV1 > 65 - 80\%), n (\%) & & & $46(65)$ & \\
\hline CLAD 2 (FEV1 > $50-65 \%), n(\%)$ & & & $17(24)$ & \\
\hline CLAD 3 (FEV1 > 35 - 50\%), n (\%) & & & $6(8)$ & \\
\hline CLAD 4 (FEV1 $\leq 35 \%), \mathrm{n}(\%)$ & & & $2(3)$ & \\
\hline \multicolumn{5}{|l|}{ CLAD phenotype according to spirometry } \\
\hline BOS (FVC decline to $>80 \%$ of baseline at CLAD onset), $n(\%)$ & & & $53(75)$ & \\
\hline RAS (FVC decline to $\leq 80 \%$ of baseline at CLAD onset), $n(\%)$ & & & $18(25)$ & \\
\hline \multicolumn{5}{|l|}{ Pulmonary function parameters at baseline and CLAD onset } \\
\hline Best postoperative FEV1 (baseline) in I, mean ( \pm SD) & $2.93 \pm 0.89$ & $2.99 \pm 0.90$ & $2.86 \pm 0.88$ & 0.34 \\
\hline FEV1 at CLAD onset in I, mean ( \pm SD) & & & $1.91 \pm 0.71$ & \\
\hline FEV1 change (baseline-CLAD onset) in I, mean ( \pm SD) & & & $-0.95 \pm 0.47$ & \\
\hline FEV1 at CLAD onset (\% of baseline) & & & $67 \pm 13$ & \\
\hline Best postoperative FVC in I, mean ( \pm SD) & $3.66 \pm 1.03$ & $3.71 \pm 1.01$ & $3.57 \pm 1.07$ & 0.35 \\
\hline FVC at CLAD onset in I, mean ( \pm SD) & & & $2.94 \pm 0.96$ & \\
\hline FVC change (baseline-CLAD onset) in I, mean ( \pm SD) & & & $-0.63 \pm 0.55$ & \\
\hline FVC at CLAD onset (\% of baseline) & & & $83 \pm 13$ & \\
\hline
\end{tabular}

Data expressed as $\mathrm{n}$ (\% of the corresponding patient group) and analyzed by Fisher's Exact Test for categoric variables, or expressed as mean \pm SD (standard deviation) or median (IQR, interquartile range), and analyze by Student's t-test for parametric continuous variables.

BOS: bronchiolitis obliterans syndrome; CLAD: chronic lung allograft dysfunction; FEV1: forced expiratory volume in 1 second; FVC: forced vital capacity; PFT: pulmonary function test; RAS: restrictive allograft syndrome. 
Table 3. Outcome of CLAD cohort patients

\begin{tabular}{|c|c|c|c|c|}
\hline Variables & $\begin{array}{c}\text { All patients } \\
\mathrm{n}=167\end{array}$ & $\begin{array}{c}\text { No CLAD } \\
n=96(57 \%)\end{array}$ & $\begin{array}{c}\text { CLAD } \\
\mathrm{n}=71(43 \%) \\
\end{array}$ & $\begin{array}{l}\text { p-value } \\
\text { No CLAD } \\
\text { vs CLAD }\end{array}$ \\
\hline \multicolumn{5}{|l|}{ Outcome } \\
\hline Follow-up days after transplant, median (range) & $2189(468-5125)$ & & & \\
\hline Dead, n (\%) & $37(22)$ & $12(13)$ & $25(35)$ & 0.001 \\
\hline Re-transplanted, n (\%) & $8(5)$ & $0(0)$ & $8(11)$ & 0.001 \\
\hline Primary cause of death & & & & $<0.001$ \\
\hline Infection, $\mathrm{n}(\%)$ & $4(11)$ & $2(17)$ & $2(8)$ & \\
\hline Malignancy, n (\%) & $6(16)$ & $5(42)$ & $1(4)$ & \\
\hline Cardiovascular, n (\%) & $2(5)$ & $1(8)$ & $1(4)$ & \\
\hline Cerebrovascular, n (\%) & $1(3)$ & $1(8)$ & $0(0)$ & \\
\hline Rejection, $\mathrm{n}(\%)$ & $0(0)$ & $0(0)$ & $0(0)$ & \\
\hline CLAD, n (\%) & $20(54)$ & $0(0)$ & $20(80)$ & \\
\hline Other, $\mathrm{n}(\%)$ & $4(11)$ & $3(25)$ & $1(4)$ & \\
\hline
\end{tabular}

Data expressed as $\mathrm{n}$ (\% of the corresponding patient group) and analyzed by Fisher's Exact Test for categoric variables, or expressed as median (range) for parametric continuous variables.

CLAD: chronic lung allograft dysfunction. 
Table 4. Univariate Cox regression analysis on graft loss of CLAD cohort patients

\begin{tabular}{|c|c|c|c|}
\hline Covariate & Hazard ratio & 95\% Confidence interval & $\mathrm{p}$-value \\
\hline CLAD & 2.91 & 1.49 to 5.67 & 0.002 \\
\hline Single lung transplant & 2.08 & 0.73 to 5.95 & 0.17 \\
\hline Preoperative ECMO & 1.67 & 0.59 to 4.73 & 0.33 \\
\hline Preoperative mechanical ventilation & 1.80 & 0.64 to 5.09 & 0.27 \\
\hline Postoperative ECMO & 20.31 & 0 to $3.12 \mathrm{E}+10$ & 0.78 \\
\hline Best postoperative FEV1 & 0.58 & 0.41 to 0.83 & 0.003 \\
\hline Best postoperative FVC & 0.59 & 0.43 to 0.83 & 0.002 \\
\hline Recipient age & 1.01 & 0.98 to 1.04 & 0.48 \\
\hline Recipient sex & 1.06 & 0.58 to 1.94 & 0.86 \\
\hline Recipient history of smoking & 1.30 & 0.70 to 2.42 & 0.40 \\
\hline Primary diagnosis & & & 0.26 \\
\hline CMV D+/R- & 2.60 & 1.19 to 5.69 & 0.02 \\
\hline Transplant urgency & & & 0.54 \\
\hline Donor age & 1.02 & 1.00 to 1.04 & 0.10 \\
\hline Donor sex & 1.36 & 0.75 to 2.47 & 0.32 \\
\hline Extended criteria donor & 1.53 & 0.83 to 2.83 & 0.17 \\
\hline Total ischemia time & 1.00 & 1.00 to 1.01 & 0.45 \\
\hline \multicolumn{4}{|l|}{ More than 4 HLA mismatches in HLA-A, B or } \\
\hline DR & 0.76 & 0.41 to 1.41 & 0.38 \\
\hline PRA positive before transplantation & 2.00 & 1.06 to 3.75 & 0.03 \\
\hline CsA as primary immunosuppression & 2.00 & 0.48 to 8.33 & 0.34 \\
\hline Tacrolimus as primary immunosuppression & 0.50 & 0.12 to 2.08 & 0.34 \\
\hline \multicolumn{4}{|l|}{ Biopsy-proven rejections } \\
\hline Any abnormal finding $<1$ year & 1.32 & 0.71 to 2.44 & 0.38 \\
\hline Any acute rejection $<1$ year & 1.21 & 0.66 to 2.22 & 0.53 \\
\hline$\geq \mathrm{A} 2$ grade $<1$ year & 1.10 & 0.58 to 2.12 & 0.77 \\
\hline Any airway inflammation $<1$ year & 1.48 & 0.76 to 2.88 & 0.26 \\
\hline$\geq \mathrm{B} 2$ grade $<1$ year & 0.05 & 0.00 to 14.97 & 0.30 \\
\hline
\end{tabular}

CLAD: chronic lung allograft dysfunction; CMV: cytomegalovirus; CSA: Cyslosporine A; D: donor; ECMO: extra-corporeal membrane oxygenation; FEV1: forced expiratory volume in 1 second; FVC: forced vital; HLA: human leukocyte antigen; PRA: panel reactive antibody; R: Recipient. 
Table 5. Multivariate Cox regression analysis on graft loss of CLAD cohort patients

\begin{tabular}{lccc}
\hline Covariate & Hazard ratio & 95\% Confidence interval & p-value \\
\hline CLAD & 2.76 & 1.36 to 5.60 & 0.005 \\
Single lung transplant & 1.20 & 0.31 to 4.60 & 0.80 \\
Best postoperative FEV1 & 1.27 & 0.45 to 3.57 & 0.65 \\
Best postoperative FVC & 0.60 & 0.24 to 1.49 & 0.27 \\
CMV D+/R- & 1.84 & 0.77 to 4.40 & 0.17 \\
Donor age & 1.01 & 0.98 to 1.04 & 0.40 \\
Extended criteria donor & 1.19 & 0.57 to 2.48 & 0.65 \\
PRA positive before transplantation & 1.82 & 0.91 to 3.67 & 0.09 \\
\hline
\end{tabular}

All covariates with $p$-value of $<0.20$ in univariate Cox regression analysis were included in multivariate Cox regression analysis.

CLAD: chronic lung allograft dysfunction; CMV: cytomegalovirus; D: Donor; FEV1: forced expiratory volume in 1 second; FVC: forced vital capacity; PRA: panel reactive antibody; R: Recipient. 


\section{Figures captions}

Figure 1. Flow-chart of the study. Adult de novo lung transplant patients with adequate pulmonary function parameters available were included in the CLAD patient cohort. CLAD was defined by persistent pulmonary function decline without other specific causes. CLAD: chronic allograft dysfunction; FEV1: forced expiratory volume in 1 second; FVC: forced vital capacity.

Figure 2. Recipient and graft survivals and effect of CLAD. Recipient survival, graft survival, and CLAD and re-transplant -free survival (A) of all adult de novo lung transplantations performed between January 2003 and December 2015 (n=175). Recipient (B) and graft survival (C) of CLAD cohort patients $(n=167)$ stratified by the presence of CLAD. Recipient (D) and graft survival (E) after CLAD onset $(n=71)$ stratified by CLAD stage. Graft survival after CLAD onset in BOS and RAS patients $(\mathrm{F})$ with the CLAD phenotype determined by spirometric criteria (FVC decline to $\leq 80 \%$ of baseline at CLAD onset results in RAS classification). Kaplan-Meier survival curves and log-rank test. Kaplan-Meier survival curves extended up to 10 years (A-C) or 7 years (D-F) as number of patients at risk becomes small. Kaplan-Meier survival curves for recipient survival, graft survival, and CLAD and re-transplant -free survival of the same cohort depicted in panel A. BOS: bronchiolitis obliterans syndrome; CLAD: chronic lung allograft dysfunction; FVC: forced vital capacity: RAS: restrictive allograft syndrome. 
Figure 1

$141 \times 149 \mathrm{~mm}(600 \times 600 \mathrm{DPI})$ 

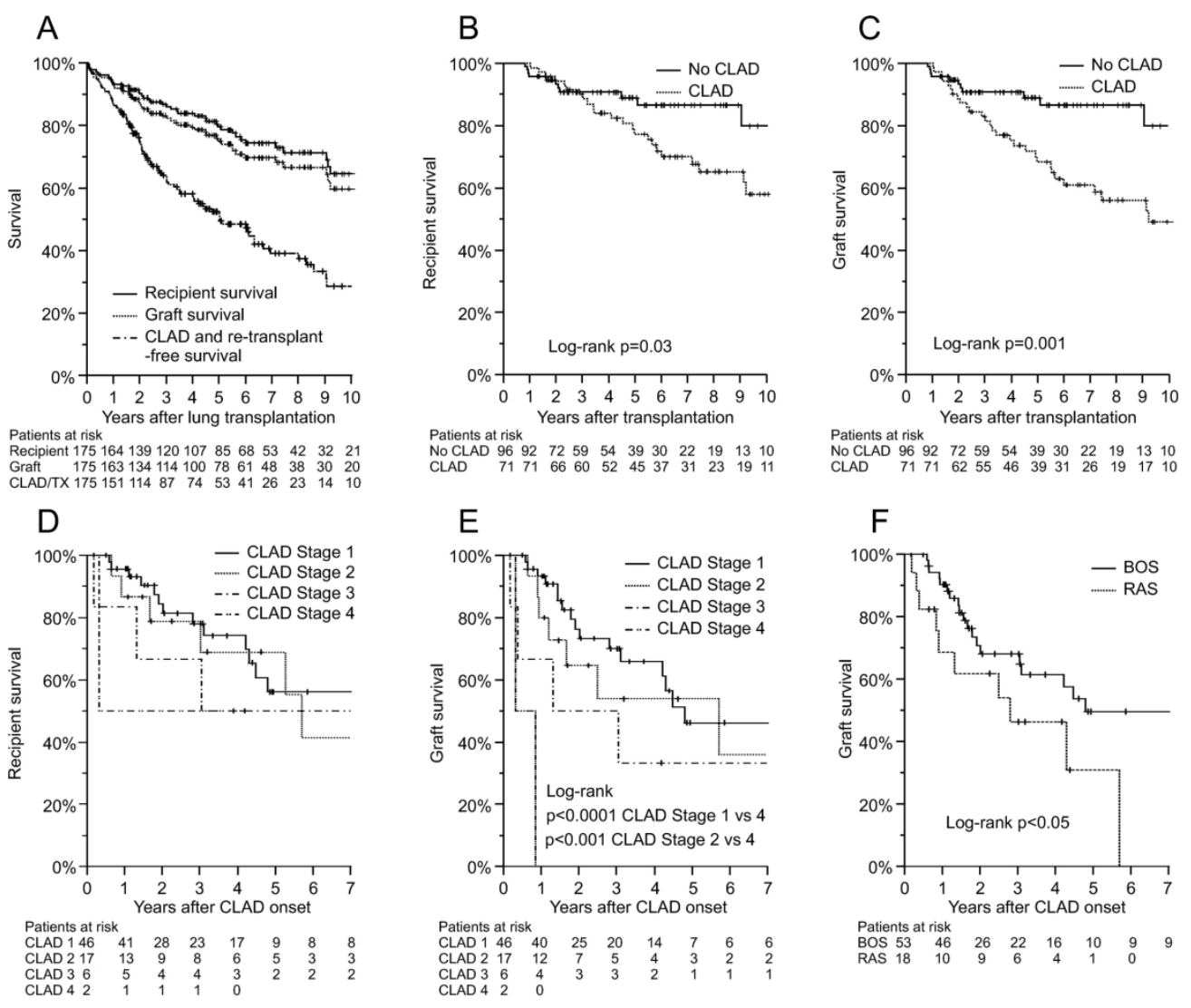

Figure 2

$204 \times 171 \mathrm{~mm}(300 \times 300$ DPI)

http://mc.manuscriptcentral.com/scar Email: ICDV-peerreview@journals.tandf.co.uk 


\title{
Incidence and impact of chronic lung allograft dysfunction
}

\section{after lung transplantation - single-center 14-year experience}

\author{
Antti Nykänen, Peter Raivio ${ }^{a}$, Laura Peräkyläa, Christoffer Stark ${ }^{\mathrm{a}}$, Antti Huuskonen ${ }^{\mathrm{a}}$, Karl \\ Lemström $^{\mathrm{a}}$, Maija Halme ${ }^{\mathrm{b}}$, Pekka Hämmäinen ${ }^{\mathrm{a}}$ \\ Affiliations

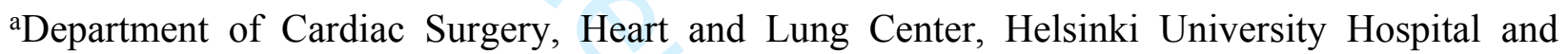 \\ University of Helsinki, Helsinki, Finland; bDepartment of Pulmonology, Heart and Lung Center, \\ Helsinki University Hospital and University of Helsinki, Helsinki, Finland
}

Correspondence to Antti Nykänen, Meilahti Tower Hospital, Haartmaninkatu 4, PL 340, 00029 Helsinki, Finland. Email: Antti.Nykanen@Hus.Fi

Total word count: 5244 


\section{Abstract (250/250 words)}

Objectives. Lung transplantation remains the only available treatment option for many end-stage lung diseases. We evaluated our long-term lung transplantation results, and the incidence and impact of chronic lung allograft dysfunction (CLAD). Design. All adult de novo lung transplants performed between 2003 and $2015(n=175)$ in a nationwide single transplant center were retrospectively analyzed. Patients surviving at least 90 days with adequate pulmonary function tests available were evaluated for CLAD ( $\mathrm{n}=167$, CLAD cohort). Kaplan-Meier survival and Cox regression analysis were used to evaluate the effect of CLAD. Results. Recipient and graft 1-, 5and 10-year survival estimates for all adult de novo lung transplants were $94 \%, 79 \%$ and $64 \%$, and $93 \%, 75 \%$ and $59 \%$, respectively. CLAD affected $43 \%$ of CLAD cohort patients, was diagnosed at a median of 2.3 years after transplantation, and impaired recipient $(\mathrm{p}=0.03)$ and graft survival $(p=0.001)$ with the most advanced CLAD stage, and restrictive CLAD phenotype, resulting in worst graft survival. CLAD was the primary cause of death in $54 \%$ of all patients, and in $80 \%$ of patients with an established CLAD diagnosis. CLAD, high-risk cytomegalovirus serostatus, and recipient preoperative sensitization increased graft loss hazard ratio in univariate regression analysis. CLAD was the only significant investigated risk factor for graft loss in multivariate regression analysis (hazard ratio $2.77,95 \%$ confidence interval 1.37-5.61, $\mathrm{p}=0.005$ ). Conclusions. Although very favourable lung transplant patient long-term survival was achieved, CLAD significantly impaired recipient and graft survival. Identification of risk factors and therapeutic options for CLAD may further improve lung transplantation results.

Keywords: Lung transplantation, end-stage pulmonary disease, chronic lung allograft dysfunction, chronic rejection, bronchiolitis obliterans syndrome 


\section{Introduction}

Lung transplantation is an established and often the only available treatment modality for endstage lung disease. The number of lung transplants performed world-wide has been increasing over time with over 4500 adult lung transplantations reported to the International Society for Heart and Lung Transplantation (ISHLT) registry for the year 2016 [1]. Lung transplant results have also gradually improved, and in the ISHLT registry, the median survival of recipients transplanted between 2009 and 6/2016 is 6.5 years [1]. Although short-term results have improved significantly, long-term survival of lung transplant recipients remains suboptimal, and lower than that of other solid organ transplant recipients $[2,3]$.

Chronic lung allograft dysfunction (CLAD) has been identified as the main reason for graft loss 1 year after lung transplantation and it affects $41 \%$ of lung transplant recipients within 5 years [1]. CLAD has been previously known as chronic rejection or bronchiolitis obliterans syndrome (BOS) [4]. The latest consensus statement defines CLAD as a persistent and chronic decline in the function of the transplanted lung in the absence of other specific reasons, and identifies separate CLAD stages according to the degree of lung function decline [5]. Reflecting the evolving knowledge of CLAD, it is currently divided into two main subgroups - BOS that results in an obstructive lung function and restrictive allograft syndrome (RAS) that is characterised by restrictive lung function [5-7]. Diagnosis of CLAD requires persistent forced expiratory volume in 1 second (FEV1) decline to $<80 \%$ of the best postoperative (baseline) FEV1. The golden standard to differentiate BOS and RAS is total lung capacity (TLC) change measured by body plethysmography [5]. Detection of restrictive lung physiology with spirometry can be used for 
CLAD phenotyping if TLC is not available [6,7]. Preventive and therapeutic options for CLAD are currently limited, but re-transplantation may be an option in selective cases [4].

\begin{abstract}
We retrospectively evaluated the long-term results of lung transplantation in our unit - a single nationwide transplant center. During the entire study period a standardized protocol of triple-drug immunosuppression and antimicrobial prophylaxis was used. We also aimed to identify risk factors for graft loss, and specifically focused on the clinical impact of CLAD. Subtypes of CLAD were evaluated by spirometric criteria [6,7] as TLC measurement with body plethysmography was not routinely used during the study period.
\end{abstract}




\section{Materials and methods}

\section{Study design and population}

We retrospectively analyzed all consecutive 175 adult (recipient $>18$ years) de novo lung transplantations performed in a nationwide single organ transplant center (Helsinki University Hospital, Helsinki, Finland) between January 2003 and December 2015. Patients were followed up until March 2017, and the median follow-up time was 6.0 years (2189 days), ranging from 1.3 to 14.0 years. Graft and recipient survival was determined for the whole study population $(\mathrm{n}=175)$, and patients who survived for at least 90 days, and had adequate pulmonary function tests available, were evaluated for CLAD ( $\mathrm{n}=167$, CLAD patient cohort). Patient information was collected from electronic patient records, and from donor and recipient data submitted to the Collaborative Transplant Study Registry [3]. The study was reviewed and approved by The Institutional Review Board of the Helsinki University Hospital.

\section{Surgical and patient management factors}

Almost all transplantations were performed through a clamshell incision (96\%) using cardiopulmonary bypass $(99 \%)$ and were bilateral lung transplants (97\%). Perioperative and postoperative management was guided by a standardized written institutional protocol. Standard triple-drug primary immunosuppression consisted of a calcineurin inhibitor (100\% of patients), mycophenolate mofetil ( $98 \%$ of patients), and corticosteroids ( $98 \%$ of patients), and only $1 \%$ of patients received induction therapy. Standard perioperative antimicrobial prophylaxis consisted of intravenous ceftazidime or meropenem, and caspofungin, and postoperative inhalation of nebulized amphotericin B. Antiviral prophylaxis was achieved with acyclovir (3 months for 
cytomegalovirus donor-/recipient-) or valganciclovir (6 months for CMV D+/R+ and D-/R+, 12 months for CMV D+/R-). Trimethoprim/sulfamethoxazole was the primary prophylaxis for pneumocystis jirovecii. Recipients routinely received proton-pump inhibitors. Azithromycin was started for patients with early signs of decreased pulmonary function or with BOS risk factors such as recurrent acute rejections, BAL neutrophilia or gastroesophageal reflux. All CLAD patients received azithromycin. The median for azithromycin initiation was 1.1 years after transplantation. Fifty-nine percent of CLAD patients were treated with azithromycin before CLAD diagnosis whereas $41 \%$ of CLAD patients were initiated with azithromycin only after CLAD diagnosis. Transbronchial surveillance biopsies were obtained 1, 2, 4, 6, 9 and 12 months after transplantation, and graded according to ISHLT classification [8]. Bronchoalveolar lavage sample and spirometry were performed during the same intervals, and yearly thereafter. TLC measurement with body plethysmography was not routinely used during the study period. Donor, recipient, and operative characteristics are given in Table 1. Criteria for ideal and extended donors were used as described previously [9].

\section{CLAD diagnosis and staging}

CLAD was evaluated using the lung function measurements recorded with spirometry during follow-up visits. All patients surviving $>90$ days who had pulmonary function tests available were included in CLAD analysis ( $\mathrm{n}=167$, Figure 1). CLAD was defined as a persistent FEV1 or forced vital capacity (FVC) decline to $\leq 80 \%$ of the best postoperative (baseline) values in the absence of other specific reasons for the lung function decline $[5,10]$. CLAD onset was defined to occur at the first time for the sustained pulmonary function decrease below the threshold [5]. CLAD was further divided into stages as suggested by the latest consensus document (CLAD Stage 1 FEV1 
$>65-80 \%$ FEV1 baseline; CLAD Stage 2 FEV1 >50-65\% FEV1 baseline; CLAD Stage 3 FEV1

$>35-50 \%$ FEV1 baseline; and CLAD 4 FEV1 $\leq 35 \%$ FEV1 baseline) [5]. As body plethysmography TLC measurement - the golden standard for BOS and RAS differentiation [6] was not routinely used during the study period, CLAD subtypes were evaluated by spirometric criteria for restrictive pulmonary function [7]. Patients whose FVC declined at CLAD onset to $\leq 80 \%$ of their baseline FVC (FVC value at the time of the best postoperative FEV1) [7] were classified as RAS, and other CLAD patients were classified as BOS.

\section{Statistical analysis}

All statistical analyses were performed using the SPSS 25.0 (IBM Corp., Armonk, NY) software. Fisher's exact test was used to compare categorical variables and Student's t-test was used to compare parametric continuous variables. For survival analyses, data is presented as Kaplan-Meier curves and analyzed with the log-rank test. $\mathrm{P}<0.05$ was considered statistically significant. Cox univariate regression analysis was used to determine the hazard ratio of covariates for graft survival. All covariates with a p-value $<0.20$ in univariate analysis were included in a Cox multivariate regression analysis. 


\section{Results}

\section{Graft and recipient survival of all de novo lung transplants}

One-hundred-seventy-five adult de novo lung transplantations were performed between January 2003 and December 2015, and pulmonary function data were available for CLAD determination in 167 patients (Figure 1). Recipient and graft 1-, 5- and 10-year survival estimates for all adult de novo lung transplants $(n=175)$ were $94 \%, 79 \%$, and $64 \%$, and $93 \%, 75 \%$, and $59 \%$, respectively (Figure 2A). In contrast, CLAD and re-transplantation -free survival estimates (survival with a well-functioning primary graft) for all adult de novo lung transplants were $86 \%, 52 \%$, and $28 \%$ for 1,5 , and 10 years, respectively (Figure 2A).

\section{CLAD diagnosis}

One-hundred-sixty-seven patients survived at least 90 days and had adequate pulmonary function measurements for the determination of possible CLAD (Figure 1, $\mathrm{n}=167$ ). Baseline donor and recipient characteristics of the CLAD patient cohort are summarized in Table 1. CLAD was diagnosed in $43 \%(n=71)$ of the CLAD cohort patients with a median diagnosis at 893 days $(2.3$ years) after transplantation (Table 2). Most CLAD patients were categorized as CLAD stage 1 or 2 whereas more advanced CLAD stage was uncommon at the time of CLAD onset (Table 2). CLAD was subclassified as BOS in $75 \%(n=53)$ and as RAS in $25 \%(n=18)$ using spirometric criteria for restrictive pulmonary function (Table 2).

\section{Effect of CLAD on outcome}


CLAD was the primary cause of death in $54 \%$ of the whole CLAD patient cohort, and in $80 \%$ of patients diagnosed with CLAD (Table 3). The most common primary cause of death in non-CLAD patients was malignancy (42\%). Eight patients (5\% of all CLAD cohort patients, $11 \%$ of patients with CLAD diagnosis) were re-transplanted due to CLAD. Recipient ( $p=0.03$, Figure $2 \mathrm{~B})$ and graft survival ( $\mathrm{p}=0.001$, Figure $2 \mathrm{C}$ ) was significantly impaired in patients with CLAD, and patients with the most advanced CLAD stage at CLAD onset had the worst graft survival (Figure 2E). Patients with RAS phenotype had worse graft survival than patients with BOS phenotype $(\mathrm{p}<0.05$, Figure $2 \mathrm{~F})$.

\section{Univariate regression analysis on graft loss}

CLAD significantly increased the hazard ratio for graft loss in a univariate regression analysis $(p=0.002$, Table 4). Other significant covariates for graft loss were high risk CMV serostatus $(\mathrm{CMV} D+/ \mathrm{R}-, \mathrm{p}=0.02)$ and recipient preoperative sensitization $(\mathrm{p}=0.03)$, whereas increased donor age $(p=0.10)$, single lung transplant $(p=0.17)$ and extended criteria donor $(p=0.17)$ were associated only with a trend towards increased hazard ratio for graft loss. Higher best postoperative FEV1 $(p=0.003)$ and FVC $(p=0.002)$ values had a significantly decreased hazard ratio for graft loss.

\section{Multivariate regression analysis on graft loss}

All covariates with a p-value $<0.20$ in the univariate analysis were included in multivariate regression analysis (Table 5). Of the investigated factors, CLAD was the only covariate that significantly increased the hazard ratio for graft loss (hazard ratio $2.77,95 \%$ confidence interval $1.37-5.61, \mathrm{p}=0.005)$. 


\section{Discussion}

We retrospectively evaluated our long-term lung transplantation results. A favourable long-term recipient survival was achieved. However, CLAD was as a major obstacle for long-term survival, as it was the most common primary cause of death, CLAD significantly impaired recipient and graft survival, and it was the strongest covariate to increase the hazard ratio for graft loss in univariate and multivariate regression analysis.

\section{Comparison of lung transplant long-term results}

The 5-year survival estimate of our adult primary lung transplant cohort transplanted between 2003 and 2015 was $79 \%$. It is favourable compared to the survival of the latest transplantation cohort (transplants between 2009 and 6/2016) reported to the ISHLT registry [1]. Although caution is warranted when interpreting the long-term survival estimates, and when comparing single center data to registry data, some donor, recipient, and patient management -related reasons may explain the favourable recipient survival of our patient cohort. First, most of our recipients received a double-lung transplant and single lung transplantation was performed only for $4 \%$ of recipients. In the ISHLT registry, single lung recipients have impaired long-term survival, and about $20 \%$ of lung transplants performed are single-lung transplantations [1] which is a significantly higher proportion than in our patient cohort. However, the evidence for the superiority of bilateral lung transplantation is not completely clear, and single-lung transplantation particularly in selective cases, or as a staged strategy, may lead to good results [11]. Second, infection was the primary cause of death in only $11 \%$ of our patients whereas infection has been reported as the known cause of death in up to $36 \%$ of lung transplant recipients early (31 days - 1 year) after transplantation [1]. Also, the incidence of airway anastomosis problems was low as no anastomosis dehiscence 
was detected, no airway stents were applied, and balloon dilatation was performed in only 5 patients (3\%). Previously, we encountered a significant amount of invasive infections that were possibly related to overimmunosuppression [12]. During the present study period, antithymocyte globulin induction was not routinely administered anymore, and close calcineurin inhibitor concentration monitoring, and standardized perioperative bacterial and fungal prophylaxis, was used [12]. Therefore, the balance of immunosuppression and antimicrobial prophylaxis in our patient cohort has probably been improved. Third, eight recipients $(5 \%$ of all patients) were retransplanted due to CLAD. Re-transplantation results have improved over time [13], and most likely the outcome of our patients re-transplanted due to CLAD would have been dismal without the possibility for a second transplant. Finally, it is possible that donor and recipient characteristics of our patient cohort affected the results. However, the median age of our donors was 46 years compared to 38 years for the donors reported to the ISHLT for the year 2012 [14], and half of our donors were extended criteria donors. The age of our recipients was comparable to the ISHLT registry. Furthermore, the proportion of transplants performed for interstitial lung disease was higher ( $50 \%$ of our primary indications) than what is reported to the ISHLT [14], and patients with this indication generally have worse survival than patients transplanted for other indications. Also, as about $10 \%$ of our recipients were bridged to transplant with ECMO or invasive ventilation, it is unlikely that significant selection bias for donors or recipients explains our results.

\section{Significance of CLAD for lung transplant recipient morbidity and mortality}

CLAD has been repeatedly identified as the main obstacle for long-term survival of lung transplant recipients $[1,4,5]$, In our patient cohort, CLAD affected $43 \%$ of the recipients and was the primary cause of death in $54 \%$ of the patients. It significantly impaired recipient and graft survival, and 
resulted in re-transplantation in $11 \%$ of the patients who had developed CLAD. Moreover, although 10-year recipient survival for all adult de novo transplants was $64 \%$, only $24 \%$ of the recipients were alive without re-transplantation, and with a well-functioning primary lung graft at 10 years. Therefore, CLAD was clearly the main cause of morbidity and mortality also in our patient cohort.

\section{Survival after CLAD onset}

Although the clinical impact of CLAD was clear, the median survival of patients affected with CLAD was 7.0 years after the onset of the disease which is longer than what is generally reported for BOS (3-5 years) or RAS (1-1.5 years) [4]. As our CLAD incidence was very similar to the ISHLT registry [1], it is possible that some factors protected against the detrimental effects of CLAD in our cohort. Our preference for double-lung transplantations may have increased the pulmonary reserve once CLAD occurred [15], and re-transplantations most probably improved survival of patients affected by CLAD substantially. Also, all CLAD patients were treated with azithromycin which may have slowed down the pulmonary function decline [16].

\section{CLAD subtypes}

Although significant advances have been made in characterizing CLAD, the pathophysiological mechanisms are not entirely understood, and preventive and treatment options currently are limited. Importantly, specific CLAD subtypes - BOS and RAS - have been identified. RAS has an even worse clinical outcome than BOS with a median survival limited to only 6-18 months after diagnosis. Despite the dismal clinical course of RAS, information on predisposing factors, 
pathogenesis and clinical course is currently very limited [17]. It is possible that detailed characterization of CLAD phenotypes may result in tailored preventive and therapeutic options. We also found that patients with restrictive CLAD phenotype had worse graft survival than patients with obstructive phenotype. As TLC measurement with body plethysmography - the golden standard also endorsed by the latest CLAD and RAS consensus statements $[5,6]$ - was not routinely used during the study period, we used spirometric criteria described by Todd et al. to differentiate BOS and RAS [7].

\section{CMV and recipient sensitization as risk factors for graft loss}

In addition to CLAD, we found that high risk CMV serostatus, and recipient preoperative sensitization, both increased the hazard ratio for graft loss in univariate analysis. CMV D+/Rserotype is a known risk factor for early and late mortality [1]. However, prophylaxis for the highrisk serotypes with 12-month valganciclovir similar to our study cohort, followed by close CMV monitoring with pre-emptive treatment strategies, reduces the incidence of CMV disease [18]. Higher preoperative PRA percentage is also an established risk factor for mortality [1] but tailored perioperative desensitization strategies may be used to safely transplant even highly sensitized recipients [19].

\section{Limitations of the current study}

Several limitations of the study must be considered when interpreting the results. Due to the retrospective nature of the study, it is possible that changes in clinical practise have occurred over time, and differences in donor and recipient characteristic, and transplant volume, over time may 
bias the incidence of time-dependent events. These likely include increased availability and use of ECMO, and more liberal donor and recipient criteria over time. Similarly, results from a singlecenter transplant center with a confined population area may be difficult to generalize. This generalization may be also affected by the transplant indication profile of our patient cohort as the proportion of ILD patients is higher, and the proportion of chronic obstructive pulmonary disease or cystic fibrosis is lower, than what is reported to the ISHLT registry [1]. Finally, due to reasons described above, we evaluated CLAD subtypes by spirometry, and not TLC measurement which is presently recommended to differentiate BOS and RAS [6].

In conclusion, retrospective analysis of our lung transplantation results demonstrates favourable long-term patient survival. Importantly, CLAD was the most significant risk factor for impaired recipient and graft survival. Therefore, in the future, identification of risk factors of CLAD and development of therapeutic options for CLAD may further improve lung transplantation results. 


\section{Acknowledgements}

We are grateful for the invaluable help of transplant coordinators Marja-Liisa Hellstedt, Catharina Yesil, and Sini Puputti, in patient management and data collection.

\section{Funding details}

This work was supported by the The Jalmari and Rauha Ahokas Foundation and The Sigrid Juselius Foundation.

\section{Declaration of interest statement}

The authors report no conflict of interest. 


\section{References}

1. Chambers DC, Cherikh WS, Goldfarb SB, et al. The International Thoracic Organ Transplant Registry of the International Society for Heart and Lung Transplantation: Thirty-fifth adult lung and heart-lung transplant report-2018; Focus theme: Multiorgan Transplantation. J Heart Lung Transplant. 2018 Oct;37(10):1169-1183.

2. Lodhi SA, Lamb KE, Meier-Kriesche HU. Solid organ allograft survival improvement in the United States: the long-term does not mirror the dramatic short-term success. Am J Transplant. 2011 Jun;11(6):1226-35.

3. Opelz G, Dohler B, Ruhenstroth A, et al. The collaborative transplant study registry. Transplant Rev (Orlando). 2013 Apr;27(2):43-5.

4. Verleden SE, Vos R, Verleden GM. Chronic lung allograft dysfunction: light at the end of the tunnel? Current opinion in organ transplantation. 2019 Jun;24(3):318-323.

5. Verleden GM, Glanville AR, Lease ED, et al. Chronic lung allograft dysfunction: Definition, diagnostic criteria, and approaches to treatment-A consensus report from the Pulmonary Council of the ISHLT. J Heart Lung Transplant. 2019 May;38(5):493-503.

6. Glanville AR, Verleden GM, Todd JL, et al. Chronic lung allograft dysfunction: Definition and update of restrictive allograft syndrome-A consensus report from the Pulmonary Council of the ISHLT. J Heart Lung Transplant. 2019 May;38(5):483-492.

7. Todd JL, Jain R, Pavlisko EN, et al. Impact of forced vital capacity loss on survival after the onset of chronic lung allograft dysfunction. Am J Respir Crit Care Med. 2014 Jan 15;189(2):159-66.

8. Stewart S, Fishbein MC, Snell GI, et al. Revision of the 1996 working formulation for the standardization of nomenclature in the diagnosis of lung rejection. J Heart Lung Transplant. 2007 Dec;26(12):1229-42.

9. Orens JB, Boehler A, de Perrot $\mathrm{M}$, et al. A review of lung transplant donor acceptability criteria. J Heart Lung Transplant. 2003 Nov;22(11):1183-200.

10. Verleden GM, Raghu G, Meyer KC, et al. A new classification system for chronic lung allograft dysfunction. J Heart Lung Transplant. 2014 Feb;33(2):127-33.

11. Subramanian MP, Meyers BF. Bilateral versus single lung transplantation: are two lungs better than one? Journal of thoracic disease. $2018 \mathrm{Jul} ; 10$ (7):4588-4601.

12. Eriksson $\mathrm{M}$, Lemstrom $\mathrm{K}$, Suojaranta-Ylinen $\mathrm{R}$, et al. Control of early Aspergillus mortality after lung transplantation: outcome and risk factors. Transplant Proc. 2010 Dec;42(10):4459-64.

13. Lindstedt $\mathrm{S}$, Dellgren $\mathrm{G}$, Iversen $\mathrm{M}$, et al. Pulmonary retransplantation in the Nordic countries. The Annals of thoracic surgery. 2015 May;99(5):1781-7.

14. Yusen RD, Christie JD, Edwards LB, et al. The Registry of the International Society for Heart and Lung Transplantation: Thirtieth Adult Lung and Heart-Lung Transplant Report--2013; focus theme: age. J Heart Lung Transplant. 2013 Oct;32(10):965-78.

15. Mason DP, Rajeswaran J, Li L, et al. Effect of changes in postoperative spirometry on survival after lung transplantation. J Thorac Cardiovasc Surg. 2012 Jul;144(1):197-203.

16. Corris PA, Ryan VA, Small T, et al. A randomised controlled trial of azithromycin therapy in bronchiolitis obliterans syndrome (BOS) post lung transplantation. Thorax. 2015 May;70(5):44250.

17. Verleden SE, Ruttens D, Vandermeulen E, et al. Predictors of survival in restrictive chronic lung allograft dysfunction after lung transplantation. J Heart Lung Transplant. 2016 Apr 16.

18. Chang A, Musk M, Lavender $\mathrm{M}$, et al. Cytomegalovirus viremia in lung transplantation during and after prophylaxis. Transpl Infect Dis. 2019 Jun;21(3):e13069. 
19. Tinckam KJ, Keshavjee S, Chaparro C, et al. Survival in sensitized lung transplant recipients with perioperative desensitization. Am J Transplant. 2015 Feb;15(2):417-26. 
Table 1. CLAD cohort characteristics.

\begin{tabular}{|c|c|c|c|c|}
\hline Variables & $\begin{array}{c}\text { All patients } \\
n=167\end{array}$ & $\begin{array}{c}\text { No CLAD } \\
n=96(57 \%)\end{array}$ & $\begin{array}{c}\text { CLAD } \\
n=71(43 \%)\end{array}$ & $\begin{array}{l}\text { p-value } \\
\text { No CLAD } \\
\text { vs CLAD }\end{array}$ \\
\hline \multicolumn{5}{|l|}{ Transplant information } \\
\hline Bilateral lung transplant, n (\%) & $161(96)$ & $95(99)$ & $66(93)$ & 0.08 \\
\hline Single lung transplant, $\mathrm{n}(\%)$ & $6(4)$ & $1(1)$ & $5(7)$ & 0.08 \\
\hline \multicolumn{5}{|l|}{ Perioperative support } \\
\hline Pre-operative ECMO, n (\%) & $15(9)$ & $9(9)$ & $6(9)$ & 1 \\
\hline Preoperative invasive ventilation, $\mathrm{n}(\%)$ & $13(8)$ & $7(7)$ & $6(9)$ & 0.78 \\
\hline Postoperative ECMO, n (\%) & $1(1)$ & $0(0)$ & $1(1)$ & 0.43 \\
\hline Transplant urgency & & & & 0.25 \\
\hline Normal, n (\%) & $141(84)$ & $78(80)$ & $63(89)$ & \\
\hline Urgent, n (\%) & $11(7)$ & $9(10)$ & $2(5)$ & \\
\hline Super Urgent, n (\%) & $15(9)$ & $9(10)$ & $6(6)$ & \\
\hline Primary diagnosis & & & & 0.045 \\
\hline Emphysema/COPD, n (\%) & $27(16)$ & $12(13)$ & $15(21)$ & \\
\hline Cystic fibrosis, n (\%) & $12(7)$ & $11(11.5)$ & $1(1)$ & \\
\hline Interstitial lung disease, $\mathrm{n}(\%)$ & $84(50)$ & $50(52)$ & $34(48)$ & \\
\hline Alpha-1 -antitrypsin deficiency, n (\%) & $17(10)$ & $11(11.5)$ & $6(9)$ & \\
\hline Pulmonary hypertension, $\mathrm{n}(\%)$ & $14(9)$ & $5(5)$ & $9(12)$ & \\
\hline Other, n (\%) & $13(8)$ & $7(7)$ & $6(9)$ & \\
\hline \multicolumn{5}{|l|}{ Recipient characteristics } \\
\hline Recipient age at transplant, median (IQR) & $56(16)$ & $56(19)$ & $58(15)$ & 0.38 \\
\hline Recipient sex female, $\mathrm{n}(\%)$ & $68(41)$ & $38(40)$ & $30(42)$ & 0.75 \\
\hline Recipient history of smoking, $\mathrm{n}(\%)$ & $100(60)$ & $55(57)$ & $45(63)$ & 0.52 \\
\hline PRA positive before transplantation, $\mathrm{n}(\%)$ & $50(30)$ & $31(32)$ & $19(29)$ & 0.73 \\
\hline$\geq 4$ HLA mismatches in HLA-A, B or DR & $71(43)$ & $39(41)$ & $32(45)$ & 0.64 \\
\hline \multicolumn{5}{|l|}{ Primary immunosuppression } \\
\hline Cyclosporine A, n (\%) & $147(88)$ & $80(83)$ & $67(94)$ & 0.03 \\
\hline Tacrolimus, n (\%) & $20(12)$ & $16(17)$ & $4(6)$ & 0.03 \\
\hline Any azithromycin use after transplantation & $147(88)$ & $76(79)$ & $71(100)$ & $<0.001$ \\
\hline \multicolumn{5}{|l|}{ Donor characteristics } \\
\hline Donor age, median (IQR) & $46(21)$ & $45.5(26)$ & $47(15)$ & 0.07 \\
\hline Donor sex female, $\mathrm{n}(\%)$ & $73(44)$ & $55(57)$ & $39(55)$ & 0.88 \\
\hline Total ischemia time minutes, median (IQR) & $259(96)$ & $262(95)$ & $254(107)$ & 0.19 \\
\hline Extended criteria donor, $\mathrm{n}(\%)$ & $86(51)$ & $47(49)$ & $39(55)$ & 0.53 \\
\hline CMV D+/R-, n (\%) & $18(11)$ & $8(5)$ & $10(14)$ & 0.31 \\
\hline \multicolumn{5}{|l|}{ Biopsy-proven rejections } \\
\hline Any abnormal finding $<1$ year, $\mathrm{n}(\%)$ & $97(58)$ & $57(59)$ & $40(57)$ & 0.87 \\
\hline Any acute rejection < 1 year, $\mathrm{n}(\%)$ & $90(54)$ & $53(55)$ & $37(53)$ & 0.88 \\
\hline$\geq A 2$ grade $<1$ year, $n(\%)$ & $47(28)$ & $31(32)$ & $16(23)$ & 0.22 \\
\hline Any airway inflammation $<1$ year, $\mathrm{n}(\%)$ & $38(23)$ & $25(26)$ & $13(19)$ & 0.35 \\
\hline$\geq \mathrm{B} 2$ grade < 1 year, $\mathrm{n}(\%)$ & $8(5)$ & $7(7)$ & $1(1)$ & 0.14 \\
\hline
\end{tabular}

Data are expressed as $\mathrm{n}$ (\% of the corresponding patient group) and analyzed by Fisher's Exact Test for 
categoric variables, or expressed as median (IQR, interquartile range) and analyzed by Student's t-test for parametric continuous variables.

CLAD: chronic lung allograft dysfunction; CMV: cytomegalovirus; COPD: chronic obstructive pulmonary disease; D: donor; ECMO: extra-corporeal membrane oxygenation; HLA: human leukocyte antigen; PRA: panel reactive antibody; R: recipient. 
Table 2. Pulmonary function and CLAD of CLAD cohort patients

\begin{tabular}{|c|c|c|c|c|}
\hline Variables & $\begin{array}{c}\text { All patients } \\
\mathrm{n}=167\end{array}$ & $\begin{array}{c}\text { No CLAD } \\
n=96(57 \%)\end{array}$ & $\begin{array}{c}\text { CLAD } \\
n=71(43 \%)\end{array}$ & $\begin{array}{l}\text { p-value } \\
\text { No CLAD } \\
\text { vs CLAD }\end{array}$ \\
\hline CLAD diagnosis days after transplant, median (IQR) & & & $839(952)$ & \\
\hline \multicolumn{5}{|l|}{ CLAD stage at CLAD onset } \\
\hline CLAD 1 (FEV1 > $65-80 \%$ ), n (\%) & & & $46(65)$ & \\
\hline CLAD 2 (FEV1 > 50 - 65\%), n (\%) & & & $17(24)$ & \\
\hline CLAD 3 (FEV1 > $35-50 \%), n(\%)$ & & & $6(8)$ & \\
\hline CLAD 4 (FEV1 $\leq 35 \%), n(\%)$ & & & $2(3)$ & \\
\hline \multicolumn{5}{|l|}{ CLAD phenotype according to spirometry } \\
\hline BOS (FVC decline to $>80 \%$ of baseline at CLAD onset), $n$ (\%) & & & $53(75)$ & \\
\hline RAS (FVC decline to $\leq 80 \%$ of baseline at CLAD onset), $n$ (\%) & & & $18(25)$ & \\
\hline \multicolumn{5}{|l|}{ Pulmonary function parameters at baseline and CLAD onset } \\
\hline Best postoperative FEV1 (baseline) in I, mean ( \pm SD) & $2.93 \pm 0.89$ & $2.99 \pm 0.90$ & $2.86 \pm 0.88$ & 0.34 \\
\hline FEV1 at CLAD onset in I, mean ( \pm SD) & & & $1.91 \pm 0.71$ & \\
\hline FEV1 change (baseline-CLAD onset) in I, mean ( $\pm S D$ ) & & & $-0.95 \pm 0.47$ & \\
\hline FEV1 at CLAD onset (\% of baseline) & & & $67 \pm 13$ & \\
\hline Best postoperative FVC in I, mean ( \pm SD) & $3.66 \pm 1.03$ & $3.71 \pm 1.01$ & $3.57 \pm 1.07$ & 0.35 \\
\hline FVC at CLAD onset in I, mean ( \pm SD) & & & $2.94 \pm 0.96$ & \\
\hline FVC change (baseline-CLAD onset) in I, mean ( \pm SD) & & & $-0.63 \pm 0.55$ & \\
\hline FVC at CLAD onset (\% of baseline) & & & $83 \pm 13$ & \\
\hline
\end{tabular}

Data expressed as $\mathrm{n}$ (\% of the corresponding patient group) and analyzed by Fisher's Exact Test for categoric variables, or expressed as mean \pm SD (standard deviation) or median (IQR, interquartile range), and analyze by Student's t-test for parametric continuous variables.

BOS: bronchiolitis obliterans syndrome; CLAD: chronic lung allograft dysfunction; FEV1: forced expiratory volume in 1 second; FVC: forced vital capacity; PFT: pulmonary function test; RAS: restrictive allograft syndrome. 
Table 3. Outcome of CLAD cohort patients

\begin{tabular}{|c|c|c|c|c|}
\hline Variables & $\begin{array}{c}\text { All patients } \\
n=167\end{array}$ & $\begin{array}{c}\text { No CLAD } \\
n=96(57 \%)\end{array}$ & $\begin{array}{c}\text { CLAD } \\
\mathrm{n}=71(43 \%) \\
\end{array}$ & $\begin{array}{l}\text { p-value } \\
\text { No CLAD } \\
\text { vs CLAD }\end{array}$ \\
\hline \multicolumn{5}{|l|}{ Outcome } \\
\hline Follow-up days after transplant, median (range) & $2189(468-5125)$ & & & \\
\hline Dead, n (\%) & $37(22)$ & $12(13)$ & $25(35)$ & 0.001 \\
\hline Re-transplanted, n (\%) & $8(5)$ & $0(0)$ & $8(11)$ & 0.001 \\
\hline Primary cause of death & & & & $<0.001$ \\
\hline Infection, $\mathrm{n}(\%)$ & $4(11)$ & $2(17)$ & $2(8)$ & \\
\hline Malignancy, n (\%) & $6(16)$ & $5(42)$ & $1(4)$ & \\
\hline Cardiovascular, n (\%) & $2(5)$ & $1(8)$ & $1(4)$ & \\
\hline Cerebrovascular, n (\%) & $1(3)$ & $1(8)$ & $0(0)$ & \\
\hline Rejection, n (\%) & $0(0)$ & $0(0)$ & $0(0)$ & \\
\hline CLAD, n (\%) & $20(54)$ & $0(0)$ & $20(80)$ & \\
\hline Other, $\mathrm{n}(\%)$ & $4(11)$ & $3(25)$ & $1(4)$ & \\
\hline
\end{tabular}

Data expressed as $\mathrm{n}$ (\% of the corresponding patient group) and analyzed by Fisher's Exact Test for categoric variables, or expressed as median (range) for parametric continuous variables.

CLAD: chronic lung allograft dysfunction. 
Table 4. Univariate Cox regression analysis on graft loss of CLAD cohort patients

\begin{tabular}{|c|c|c|c|}
\hline Covariate & Hazard ratio & 95\% Confidence interval & $\mathrm{p}$-value \\
\hline CLAD & 2.91 & 1.49 to 5.67 & 0.002 \\
\hline Single lung transplant & 2.08 & 0.73 to 5.95 & 0.17 \\
\hline Preoperative ECMO & 1.67 & 0.59 to 4.73 & 0.33 \\
\hline Preoperative mechanical ventilation & 1.80 & 0.64 to 5.09 & 0.27 \\
\hline Postoperative ECMO & 20.31 & 0 to $3.12 \mathrm{E}+10$ & 0.78 \\
\hline Best postoperative FEV1 & 0.58 & 0.41 to 0.83 & 0.003 \\
\hline Best postoperative FVC & 0.59 & 0.43 to 0.83 & 0.002 \\
\hline Recipient age & 1.01 & 0.98 to 1.04 & 0.48 \\
\hline Recipient sex & 1.06 & 0.58 to 1.94 & 0.86 \\
\hline Recipient history of smoking & 1.30 & 0.70 to 2.42 & 0.40 \\
\hline Primary diagnosis & & & 0.26 \\
\hline CMV D+/R- & 2.60 & 1.19 to 5.69 & 0.02 \\
\hline Transplant urgency & & & 0.54 \\
\hline Donor age & 1.02 & 1.00 to 1.04 & 0.10 \\
\hline Donor sex & 1.36 & 0.75 to 2.47 & 0.32 \\
\hline Extended criteria donor & 1.53 & 0.83 to 2.83 & 0.17 \\
\hline Total ischemia time & 1.00 & 1.00 to 1.01 & 0.45 \\
\hline \multicolumn{4}{|l|}{ More than 4 HLA mismatches in HLA-A, B or } \\
\hline DR & 0.76 & 0.41 to 1.41 & 0.38 \\
\hline PRA positive before transplantation & 2.00 & 1.06 to 3.75 & 0.03 \\
\hline CsA as primary immunosuppression & 2.00 & 0.48 to 8.33 & 0.34 \\
\hline Tacrolimus as primary immunosuppression & 0.50 & 0.12 to 2.08 & 0.34 \\
\hline \multicolumn{4}{|l|}{ Biopsy-proven rejections } \\
\hline Any abnormal finding $<1$ year & 1.32 & 0.71 to 2.44 & 0.38 \\
\hline Any acute rejection $<1$ year & 1.21 & 0.66 to 2.22 & 0.53 \\
\hline$\geq \mathrm{A} 2$ grade $<1$ year & 1.10 & 0.58 to 2.12 & 0.77 \\
\hline Any airway inflammation $<1$ year & 1.48 & 0.76 to 2.88 & 0.26 \\
\hline$\geq \mathrm{B} 2$ grade $<1$ year & 0.05 & 0.00 to 14.97 & 0.30 \\
\hline
\end{tabular}

CLAD: chronic lung allograft dysfunction; CMV: cytomegalovirus; CSA: Cyslosporine A; D: donor; ECMO: extra-corporeal membrane oxygenation; FEV1: forced expiratory volume in 1 second; FVC: forced vital; HLA: human leukocyte antigen; PRA: panel reactive antibody; R: Recipient. 
Table 5. Multivariate Cox regression analysis on graft loss of CLAD cohort patients

\begin{tabular}{lccc}
\hline Covariate & Hazard ratio & 95\% Confidence interval & p-value \\
\hline CLAD & 2.76 & 1.36 to 5.60 & 0.005 \\
Single lung transplant & 1.20 & 0.31 to 4.60 & 0.80 \\
Best postoperative FEV1 & 1.27 & 0.45 to 3.57 & 0.65 \\
Best postoperative FVC & 0.60 & 0.24 to 1.49 & 0.27 \\
CMV D+/R- & 1.84 & 0.77 to 4.40 & 0.17 \\
Donor age & 1.01 & 0.98 to 1.04 & 0.40 \\
Extended criteria donor & 1.19 & 0.57 to 2.48 & 0.65 \\
PRA positive before transplantation & 1.82 & 0.91 to 3.67 & 0.09 \\
\hline
\end{tabular}

All covariates with $p$-value of $<0.20$ in univariate Cox regression analysis were included in multivariate Cox regression analysis.

CLAD: chronic lung allograft dysfunction; CMV: cytomegalovirus; D: Donor; FEV1: forced expiratory volume in 1 second; FVC: forced vital capacity; PRA: panel reactive antibody; R: Recipient. 


\section{Figures captions}

Figure 1. Flow-chart of the study. Adult de novo lung transplant patients with adequate pulmonary function parameters available were included in the CLAD patient cohort. CLAD was defined by persistent pulmonary function decline without other specific causes. CLAD: chronic allograft dysfunction; FEV1: forced expiratory volume in 1 second; FVC: forced vital capacity.

Figure 2. Recipient and graft survivals and effect of CLAD. Recipient survival, graft survival, and CLAD and re-transplant -free survival (A) of all adult de novo lung transplantations performed between January 2003 and December 2015 (n=175). Recipient (B) and graft survival (C) of CLAD cohort patients $(n=167)$ stratified by the presence of CLAD. Recipient (D) and graft survival (E) after CLAD onset $(n=71)$ stratified by CLAD stage. Graft survival after CLAD onset in BOS and RAS patients $(\mathrm{F})$ with the CLAD phenotype determined by spirometric criteria (FVC decline to $\leq 80 \%$ of baseline at CLAD onset results in RAS classification). Kaplan-Meier survival curves and log-rank test. Kaplan-Meier survival curves extended up to 10 years (A-C) or 7 years (D-F) as number of patients at risk becomes small. Kaplan-Meier survival curves for recipient survival, graft survival, and CLAD and re-transplant -free survival of the same cohort depicted in panel A. BOS: bronchiolitis obliterans syndrome; CLAD: chronic lung allograft dysfunction; FVC: forced vital capacity: RAS: restrictive allograft syndrome. 